\title{
Hyperintense Optic Nerve due to Diffusion Restriction: Diffusion-Weighted Imaging in Traumatic Optic Neuropathy
}

\author{
U.K. Bodanapally, K. Shanmuganathan, R.K. Shin, D. Dreizin, L. Katzman, R.P. Reddy, and D. Mascarenhas
}

\begin{abstract}
BACKGROUND AND PURPOSE: Abnormal signal intensity of the optic nerve due to diffusion restriction may be seen in traumatic optic neuropathy. In addition to evaluating optic nerve hyperintensity on diffusion-weighted imaging, we compared the group differences of $A D C$ values between the injured and uninjured contralateral nerve and identified the relation between measured ADC values and admission visual acuity.
\end{abstract}

MATERIALS AND METHODS: We retrospectively evaluated 29 patients with traumatic optic neuropathy who underwent MR imaging with DWI. Uninjured contralateral optic nerves were used as controls. Two attending radiologists, blinded to the side of injury, independently reviewed the DWI for the presence of signal-intensity abnormality and obtained ADC values after manually selecting the ROI.

RESULTS: Hyperintensity of the optic nerve was demonstrated in 8 of the 29 patients, with a sensitivity of $27.6 \%(95 \% \mathrm{Cl}, 12.8-47.2)$ and a specificity of $100 \%(95 \% \mathrm{Cl}, 87.9-100)$. ADC values were obtained in 25 patients. The mean ADC in the posterior segment of the injured nerve was significantly lower than that in the contralateral uninjured nerve (Welch ANOVA, $F=9.7, P=.003$ ). There was a moderate-tostrong correlation between low ADC values and poor visual acuity in 10 patients in whom visual acuity could be obtained at admission $(R=$ $0.7, P=.02)$. Patients with optic nerve hyperintensity presented with worse visual acuity.

CONCLUSIONS: Hyperintensity of the optic nerve due to diffusion restriction can serve as a specific imaging marker of traumatic optic neuropathy. When paired with reduced ADC values, this finding may be an important surrogate for visual acuity.

ABBREVIATIONS: $\mathrm{ON}=$ optic nerve; $\mathrm{Q}=$ quartile; $T \mathrm{ON}=$ traumatic optic neuropathy; $\mathrm{VA}=$ visual acuity

$\mathbf{T}$ raumatic optic neuropathy (TON) is an acute injury of the optic nerve $(\mathrm{ON})$, typically presenting with severe impairment of visual function. TON is often classified on the basis of the location of the injury. Anterior ON injury is usually associated with $\mathrm{ON}$ head avulsion and disruption of circulation at the $\mathrm{ON}$ head. These patients present with intraocular hemorrhage and disruption of anatomy at the ON head on funduscopy. Posterior TON involves the nerve at a site proximal to where the ophthalmic artery enters the ON. ${ }^{1}$ Clinical diagnosis of posterior TON is based on the presence of a relative afferent pupillary defect, decreased visual acuity (VA), normal funduscopic examination

Received November 3, 2014; accepted after revision December 23.

From the Departments of Radiology, R Adams Cowley Shock Trauma Center (U.K.B., K.S., D.D.) and Ophthalmology and Visual Sciences (R.K.S., L.K.), University of Maryland Medical Center, Baltimore, Maryland; Albert Einstein College of Medicine of Yeshiva University (R.P.R.), Bronx, New York; and University of Maryland School of Medicine (D.M.), Baltimore, Maryland.

Please address correspondence to Uttam K. Bodanapally, MBBS, 22 S Greene St, Department of Radiology, University of Maryland Medical Center, Baltimore, MD 21201; e-mail: ubodanapally@umm.edu

http://dx.doi.org/10.3174/ajnr.A4290 findings, and no apparent intraocular pathology. ${ }^{2-4}$ The focus of this study was limited to posterior TON. Contusion, necrosis, concussion, hemorrhage, nerve fiber tears, and infarction due to vascular thrombosis or spasm have all been implicated as potential mechanisms of TON. ${ }^{5,6}$ Specific causes include motor vehicle collisions, falls, assaults, blunt force effects of penetrating trauma, and surgical mishaps in and around the optic nerve, with a reported high prevalence among young men. ${ }^{7}$

The architecture of the orbit facilitates transfer of impact forces from facial trauma to the region of the optic canal, where the nerve is vulnerable to injury due to the shearing effect between its fixed and mobile portions. ${ }^{7-9}$ The concept of primary and secondary injury in TON has been proposed by Walsh. ${ }^{10}$ Primary injury results from immediate shearing of the retinal ganglion cell axons of the nerve. Secondary injury occurs from a complex biochemical cascade of events that follows the primary injury, resulting in edema of the $\mathrm{ON}$ within the inflexible optic canal. ${ }^{11}$ Edema of the nerve in the optic canal produces a compartment syndrome, resulting in nerve ischemia due to compression of the pial vascular plexus. $^{12,13}$ 

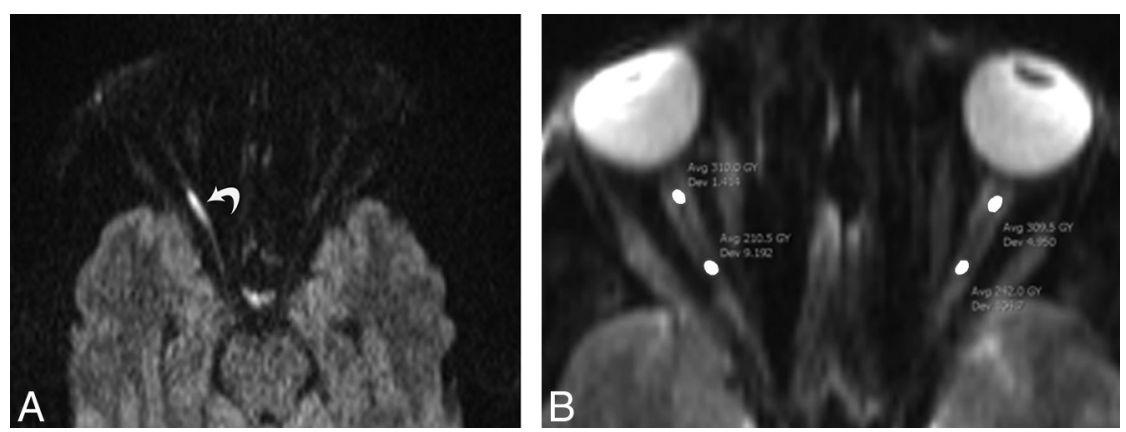

\section{MR Imaging Protocol and Image \\ Analysis}

All imaging was performed on a 1.5T Avanto scanner (Siemens, Erlangen, Germany) with parallel imaging capacity. Twenty patients underwent DWI as part of MR imaging of the brain; and in the remaining 9 patients, DWI was part of orbital MR imaging. According to our institutional protocol, DWI is performed at a $5-\mathrm{mm} \mathrm{sec-}$

FIG 1. A 37-year-old man with right-sided TON following a motor vehicle collision. Axial diffusionweighted image $(A)$ shows hyperintensity of the posterior segment of the optic nerve (curved arrow) due to restricted diffusion. $B$, Axial DWI $(b=0)$ of the same patient shows ROI placement on the anterior and posterior segments of intraorbital optic nerve.

Conventional MR imaging findings are normal in most patients with TON. ${ }^{3,5}$ An earlier study with DTI in TON showed decreased axial diffusion and ADC values in the posterior segment of the injured $\mathrm{ON}^{3}$ In this retrospective study, we aimed to determine the ability of abnormal signal intensity of the ON due to diffusion restriction on DWI to diagnose TON, compare the group differences of ADC values between the injured and uninjured contralateral nerves, and identify the relation between the measured ADC values and admission VA.

\section{MATERIALS AND METHODS}

The study was compliant with the Health Insurance Portability and Accountability Act, and permission was obtained from our institutional review board. The study was conducted at a level I trauma center. The inclusion criteria for this retrospective study were the following: 1) history of blunt craniofacial trauma between January 2004 and December 2013,2) acquisition of DWI as part of the MR imaging examination of the brain or orbits ( $\leq 15$ days after trauma), 3) 18 years of age or older, and 4) a clinically confirmed diagnosis of TON on formal ophthalmology consultation. Exclusion criteria were the following: 1) TON caused by blunt force or blast effects of penetrating trauma, and 2) abnormal funduscopy findings or traumatic structural intraocular pathology on clinical examination. During the study period, MR imaging was not routinely performed on patients with TON. MR imaging of the brain or orbits was performed at the discretion of the clinicians.

At the study institute, ophthalmology is consulted on all patients with orbital or periorbital soft-tissue trauma and craniofacial fractures, to detect injuries involving the visual system. All patients referred to ophthalmology are evaluated while in the trauma resuscitation unit. A clinical diagnosis of TON is made in the presence of decreased VA, relative afferent pupillary defect, and normal funduscopic examination findings. Testing for VA or funduscopy is usually not possible in patients who are unconscious due to sedation, mechanical ventilation, or associated head injuries. In such patients, a probable diagnosis of TON is made on the basis of the presence of a relative afferent pupillary defect, and the diagnosis is confirmed by re-evaluating the patients after they regain consciousness or after extubation. ing in 3 orthogonal directions with $b$-values of $0,500,1000$ $\mathrm{s} / \mathrm{mm}^{2}$. The TE for the DWI was $86 \mathrm{~ms}$ with a TR of $5400 \mathrm{~ms}$. A section thickness of $5 \mathrm{~mm}$ and an FOV of $220 \times 220 \mathrm{~mm}$ at an acquisition matrix of $134 \times 192$ pixels were used.

\section{Image Analysis}

The DWI was evaluated on the PACS of our institution by 2 fellowship-trained and board-certified trauma radiologists (U.K.B. and D.D.) with 8 and 3 years of experience, respectively. Images were first evaluated qualitatively and then quantitatively. For qualitative analysis, the reviewers were blinded to the side of injury and individually evaluated the ONs for bilateral or unilateral signal intensity changes at $b=1000$. The reviewers classified the signal intensity into the following categories: higher, equal, or lower than the signal intensity of the contralateral ON and brain parenchyma. In the case of increased signal intensity, the extent of involvement (posterior and anterior segments) was documented (Figs 1 and 2). The usual 20- to 25-mm intraorbital ON was divided into anterior and posterior segments at approximately 10 $\mathrm{mm}$ behind the globe. The division was made to evaluate intersegmental differences in signal intensity and ADC values because the blood supply of the posterior segment is different from that of the anterior segment and the posterior segment has a greater propensity for injury.

For quantitative analysis, the ADCs within the ON were measured by ROI analysis. For placement of an ROI, both readers were instructed to include the posterior and anterior segments of the ON separately. Elliptic ROIs of approximately $7 \mathrm{~mm}^{2}$ (range, $6-8 \mathrm{~mm}^{2}$ ) were manually drawn over the long axis of the $\mathrm{ON}$ on the spin-echo echo-planar images $(b=0)($ Fig $1 B)$ and then were transferred to the ADC maps. The anterior segment ROIs were drawn at a distance of approximately $3-4 \mathrm{~mm}$ from the globe, and the posterior ROI, at approximately $15-18 \mathrm{~mm}$ from the globe. Data from the posterior and anterior segments were analyzed separately. To avoid CSF partial volume artifacts, the ROI mostly included voxels at the nerve center. In addition, areas of susceptibility artifacts were excluded.

\section{Statistical Analysis}

The correlation between the ADC measurements of the 2 reviewers was performed by using a Pearson correlation coefficient. The sensitivity and specificity of signal-intensity abnormality of the 

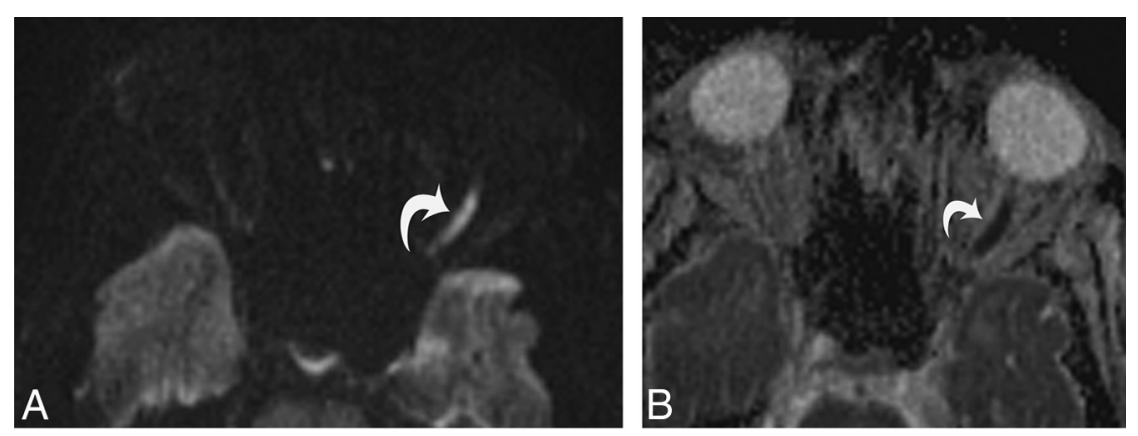

information and the clinical findings, the patient was diagnosed with iatrogenic TON. There were 17 left-sided injuries and 12 right-sided $\mathrm{ON}$ injuries. The median length from the time of trauma to imaging was 7 days $\left(\mathrm{Q}_{3}-\mathrm{Q}_{1}=\right.$ 9.5 days). Table 1 provides the data regarding the time from injury to MR imaging, VA, ON hyperintensity, and ADC values.
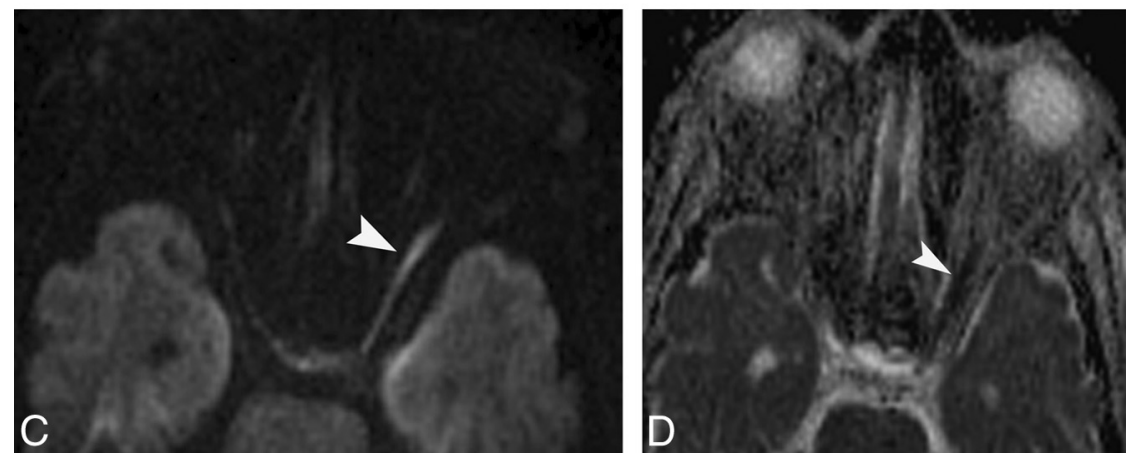

FIG 2. A 64-year-old man with left-sided TON following a fall. Axial diffusion-weighted image $(A)$ and $A D C$ map $(B)$ show concomitant extension of hyperintensity into the anterior segment of optic nerve (curved arrow), with corresponding hypointense signal on the ADC map suggestive of restricted diffusion. Posterior segment involvement on the diffusion-weighted image (C) and ADC map (D).

ON on DWI was calculated by a contingency table. The mean ADC of both reviewers was used for statistical analysis. A 1-way ANOVA was used to compare the between-group differences of ADC values. After testing for homogeneous variance (Levene test), a post hoc analysis was performed by using the Welch and Wilcoxon tests. Receiver operating characteristic curve analysis was used to evaluate the usefulness of ADC measurements. Linear regression analysis was used to determine the relationship between admission VA and ADC values. For all analysis, a $P$ value $<.05$ was considered statistically significant. Statistical analysis was performed by using JMP 11 software (SAS Institute, Cary, North Carolina).

VA was converted into a logarithm of the minimum angle of resolution units to provide a numeric scale for statistical analysis.

\section{RESULTS \\ Demographics}

A search of the trauma data base of the institution yielded 183 patients with a clinical diagnosis of TON. Twenty-nine patients (21 men, 8 women; mean age, 40.8 years; range, $18-69$ years) of the total 183 were evaluated with DWI and were retrospectively recruited for the study. All patients had unilateral TON. The mechanism of injury in patients with TON was a motor vehicle collision in 15 , assault in 6 , a fall in 4 , pedestrian struck in 3 , and orbital surgery in 1. A single patient with iatrogenic TON was included in the study. The patient developed visual impairment after simple orbital floor fracture repair without documented perioperative hypotension or elevated intraorbital pressure, thus excluding the possibility of surgical posterior ischemic optic neuropathy and orbital compartment syndrome. On the basis of the was perfect agreement between the 2 reviewers in the assessment of the signal intensity of the ONs.

\section{Quantitative Analysis}

The reviewers independently excluded 4 patients because of inaccurate ADC measurements due to susceptibility artifacts caused by the air-bone-tissue interface and/or partial volume effects, which affected the ROI placement over the ONs. The mean posterior segment $\mathrm{ADC}$ of the contralateral uninjured $\mathrm{ON}$ in the remaining 25 patients was $1.32 \times 10^{-3} \mathrm{~mm}^{2} / \mathrm{s}$ (standard error, $0.85 \times 10^{-3} \mathrm{~mm}^{2} / \mathrm{s} ; 95 \% \mathrm{CI}, 1.15 \times 10^{-3} \mathrm{~mm}^{2} / \mathrm{s}$ to $1.49 \times 10^{-3}$ $\mathrm{mm}^{2} / \mathrm{s}$ ). The mean posterior segment ADC of the injured nerve was $0.94 \times 10^{-3} \mathrm{~mm}^{2} / \mathrm{s}$ ( standard error, $0.85 \times 10^{-3} \mathrm{~mm}^{2} / \mathrm{s} ; 95 \%$ CI, $0.77 \times 10^{-3} \mathrm{~mm}^{2} / \mathrm{s}$ to $1.11 \times 10^{-3} \mathrm{~mm}^{2} / \mathrm{s}$ ) (Fig 3). The posterior segment $\mathrm{ADC}$ differed significantly between injured and uninjured nerves in Welch ANOVA analysis $(F=9.7, P=.003$ and Wilcoxon $P=.015$ ). Receiver operating characteristic curve analysis determined a discrimination ability of 0.7 (area under the curve) between the injured and uninjured contralateral nerve with optimum sensitivity and specificity at a mean ADC value of $0.92 \times 10^{-3} \mathrm{~mm}^{2} / \mathrm{s}$.

There was no statistical difference in the mean anterior segment ADC values between the injured and uninjured nerves ( $P=$ 0.24 ) (Table 2). In subsequent analysis, the patients were divided into 2 subgroups. Group 1 consisted of 14 patients with a time from injury to MR imaging examination of $\leq 7$ days, and the remaining 11 patients constituted group 2 with a time from injury to MR imaging of $>7$ days. In group 1 patients, the mean posterior segment $\mathrm{ADC}$ of the injured $\mathrm{ON}$ was significantly reduced 
Table 1: Results of the 29 cases with traumatic optic neuropathy, their respective time from injury to MRI, and visual acuity

\begin{tabular}{|c|c|c|c|c|}
\hline $\begin{array}{c}\text { Patient } \\
\text { No. }\end{array}$ & $\begin{array}{c}\text { Days } \\
\text { between } \\
\text { Injury } \\
\text { and MRI }\end{array}$ & $\begin{array}{l}\text { VA on } \\
\text { Affected } \\
\text { Side } \\
\text { (logMAR) }\end{array}$ & $\begin{array}{l}\text { Hyperintensity } \\
\text { due to } \\
\text { Diffusion } \\
\text { Restriction }\end{array}$ & $\begin{array}{c}\mathrm{ADC}\left(10^{-3} \mathrm{~mm}^{2} / \mathrm{s}\right) \\
\text { in the Posterior } \\
\text { Segment of the } \\
\text { Injured ON }\end{array}$ \\
\hline 1 & 12 & NA & - & $\mathrm{NE}$ \\
\hline 2 & 15 & NA & - & $\mathrm{NE}$ \\
\hline 3 & 1 & -0.7 & - & $\mathrm{NE}$ \\
\hline 4 & 7 & NA & - & NE \\
\hline 5 & 7 & -4.7 & + & 0.3 \\
\hline 6 & 15 & NA & - & 1.25 \\
\hline 7 & 15 & NA & - & 1.4 \\
\hline 8 & 9 & -4.7 & - & 0.825 \\
\hline 9 & 1 & -1 & - & 1.5 \\
\hline 10 & 2 & -4.7 & - & 0.85 \\
\hline 11 & 2 & -0.48 & - & 1.25 \\
\hline 12 & 12 & NA & - & 1.3 \\
\hline 13 & 10 & NA & - & 1.6 \\
\hline 14 & 7 & NA & - & 1.35 \\
\hline 15 & 5 & NA & - & 1.35 \\
\hline 16 & 8 & -4.7 & + & 0.15 \\
\hline 17 & 12 & -1 & - & 0.92 \\
\hline 18 & 11 & NA & - & 1.65 \\
\hline 19 & 12 & NA & + & 0.75 \\
\hline 20 & 1 & -4.7 & + & 0.38 \\
\hline 21 & 4 & -4.7 & + & 0.05 \\
\hline 22 & 1 & NA & - & 1.074 \\
\hline 23 & 4 & NA & - & 1.15 \\
\hline 24 & 3 & NA & + & 0.6 \\
\hline 25 & 11 & NA & - & 1.15 \\
\hline 26 & 10 & NA & - & 1.65 \\
\hline 27 & 5 & -4.7 & + & 0.15 \\
\hline 28 & 1 & NA & - & 0.65 \\
\hline 29 & 1 & NA & + & 0.285 \\
\hline
\end{tabular}

Note:- $\log$ MAR indicates logarithm of the minimum angle of resolution; NA, not available; NE, not evaluated; +, present; -, absent.

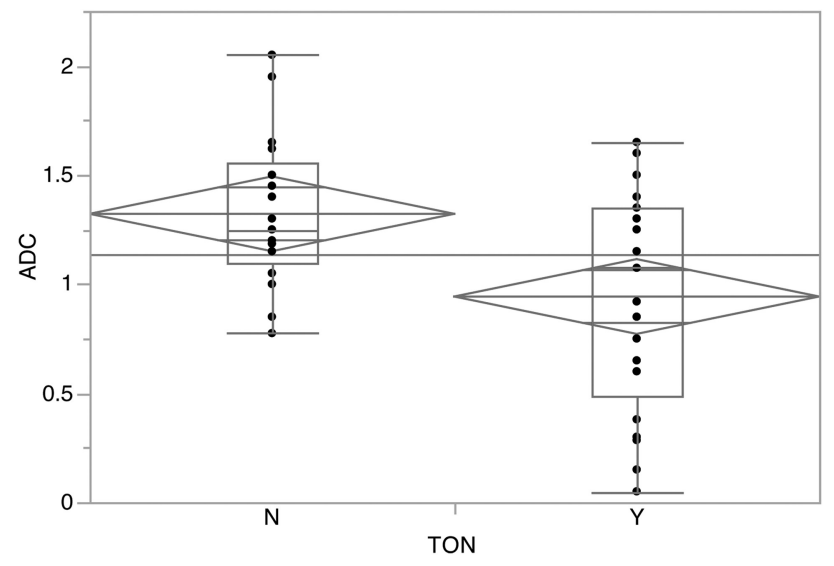

FIG 3. Graph comparing the $\operatorname{ADC}\left(10^{-3} \mathrm{~mm}^{2} / \mathrm{s}\right)$ values and the clinical diagnosis of TON. Box-and-whisker plots with lines representing medians, interquartile ranges, and greatest and least values. Mean diamond plots with horizontal lines represent mean and upper and lower $95 \%$ confidence points for each group.

relative to the contralateral posterior $\mathrm{ON}(P=.03)$. The ADC reduction did not reach statistical significance in group 2 patients $(P=.17)$. The interrater reliability in measuring the ADC of the ONs was very good, with a Pearson correlation coefficient of 0.87 (95\% CI, 0.79-0.92).

Admission VA was available in 10 of the 29 patients. Admission VA could not be obtained in 19 patients due to associated injuries for which patients were under sedation, on mechanical ventilation, or under the influence of other mind-altering drugs. There was a moderate-to-strong correlation between low ADC values and poor VA in the 10 patients analyzed (ie, a lower logarithm of the minimum angle of resolution) (Pearson $r=0.7, P=$ .02 ; Spearman $\rho=0.66, P=.03)$. In patients with ON hyperintensity on qualitative analysis, 5 of the 8 patients had available VA. All 5 patients presented with no light perception. The median logarithm of the minimum angle of resolution in patients with ON hyperintensity was -4.7 (no light perception) $\left(\mathrm{Q}_{1}-\mathrm{Q}_{3}=0\right.$ ); and in those without hyperintensity, the logarithm of the minimum angle of resolution was $-1(20 / 200)\left(\mathrm{Q}_{1}-\mathrm{Q}_{3}=-4, P=\right.$ $.012)$.

\section{DISCUSSION}

The results of our study indicate that hyperintensity of the ON predominantly affecting the posterior segment of the intraorbital $\mathrm{ON}$ is associated with a clinical diagnosis of TON in the appropriate clinical setting. Although a decreased ADC value of the injured nerve and alterations in other DTI parameters have been previously described, ${ }^{3}$ a qualitative visual assessment of ON hyperintensity on DWI can provide a helpful clinical indicator of TON. The sensitivity of this sign was $27.6 \%$ (95\% CI, $12.8 \%-$ $47.2 \%$ ) and the specificity was $100 \%$ (95\% CI, $87.9 \%-100 \%)$. Hence, the presence of hyperintensity is helpful in making a diagnosis of TON, but its absence should not be interpreted as an absence of TON.

Our results also showed a statistically significant difference between the mean posterior segment ADC values of the injuredversus-uninjured nerves, which can help in the discrimination of TON. In the limited number of patients with TON with available VA, there was a correlation between low ADC values and poor $\mathrm{VA}$, and those with ON hyperintensity due to diffusion restriction had the worst VA (ie, no light perception at presentation). The correlation among ON hyperintensity, low ADC values, and poor VA at admission may help clinicians predict the likelihood of visual recovery in patients with severe trauma, especially in those in whom VA could not be obtained due to various factors, because the initial VA is the strongest predictor of visual recovery. ${ }^{14-16}$ This information has the potential to help prioritize therapeutic interventions should new therapies become available.

Acute ischemia and contusions in the brain parenchyma and spinal cord present as hyperintense signal on DWI at measurements with large $b$-values, and the calculated ADC map shows decreased diffusivity. ${ }^{17,18}$ Although contusion-related necrosis and ischemia of the ON are implicated in TON at postmortem examination, the signal-intensity changes in DWI corresponding to these histologic findings have not been evaluated in patients with TON, to our knowledge. The hyperintensity of the injured ON seen in our patients, most of whom have involvement of the posterior segment, supports the hypothesis that the nerve segment that is most vulnerable to primary injury is at the level of the optic canal and that secondary injury from compartment syndrome occurs in the optic canal, causing nerve ischemia. The 2 cases with concomitant varying levels of extension of hyperintensity into the anterior segment may be explained by 
Table 2: Mean and median ADC values on injured and uninjured contralateral optic nerves in patients with traumatic optic neuropathy

\begin{tabular}{|c|c|c|c|c|c|c|}
\hline \multirow[b]{2}{*}{ ON Segment } & \multicolumn{2}{|c|}{ Injured ON } & \multicolumn{2}{|c|}{ Contralateral ON } & \multicolumn{2}{|c|}{$\begin{array}{l}P \text { Value (between Injured } \\
\text { and Contralateral Side) }\end{array}$} \\
\hline & $\begin{array}{c}\text { Mean ADC, } \\
\text { SE }\left(10^{-3} \mathrm{~mm}^{2} / \mathrm{s}\right)\end{array}$ & $\begin{array}{c}\text { Median ADC, } \\
\mathrm{Q}_{3}-\mathrm{Q}_{1}\left(10^{-3} \mathrm{~mm}^{2} / \mathrm{s}\right)\end{array}$ & $\begin{array}{c}\text { Mean ADC, } \\
\text { SE }\left(10^{-3} \mathrm{~mm}^{2} / \mathrm{s}\right)\end{array}$ & $\begin{array}{c}\text { Median ADC, } \\
\mathrm{Q}_{3}-\mathrm{Q}_{1}\left(10^{-3} \mathrm{~mm}^{2} / \mathrm{s}\right)\end{array}$ & Welch Test & Wilcoxon Test \\
\hline Posterior segment & $0.94(0.85)^{a}$ & $1.07(0.86)^{a}$ & $1.32(0.85)^{a}$ & $1.25(0.46)^{\mathrm{a}}$ & $.0033^{\mathrm{a}}$ & $.015^{\mathrm{a}}$ \\
\hline Anterior segment & $1.37(0.73)$ & $1.46(0.36)$ & $1.49(0.73)$ & $1.42(0.47)$ & .24 & .49 \\
\hline
\end{tabular}

Note:-SE indicates standard error.

${ }^{a}$ Statistically significant differences $(P<.05)$

spasm or thrombosis of the axial centrifugal vascular system formed by intraneural branches of the central retinal artery. Subanalysis of patients based on the time from injury to MR imaging examination showed a statistically significant decrease in the posterior segment ADC when the imaging was performed within 7 days after injury. The reduction in ADC did not reach statistical significance when the imaging was performed between 7 and 15 days after trauma, however. This phenomenon is most likely due to ADC normalization, though the time course of the normalization process is not known for the optic nerve in the setting of trauma.

There is limited literature regarding the utility of functional MR imaging of ON injury. An earlier study by Bodanapally et $\mathrm{al}^{3}$ evaluated the role of DTI in 12 patients with TON and reported a lower axial diffusivity in both of the segments of the ON and a decrease in ADC in the posterior segment. The decrease in ADC, however, was not statistically significant in relation to the contralateral uninjured nerve. ${ }^{3}$ The significant decrease in ADC values in our current study can be due to a larger study cohort, difference in the technique of ADC measurements, and/or the inclusion of patients with hyperintense $\mathrm{ON}$ due to diffusion restriction. In contrast to the above findings, Yang et al ${ }^{19}$ evaluated 6 patients with DTI (mean time from injury to imaging, 5.2 days) and showed an increase in mean ADC values. The authors ascribed this increase to the ischemic demyelination or necrosis of the nerve fiber bundles. Contrary to their explanation, we suggest that the acute injury of the optic nerve is manifested by a decrease in the ADC values similar to the reduced ADC values in acute cerebral and spinal cord injuries.

TON is a clinical diagnosis, but a clinical examination may not be possible in patients with severe polytrauma due to sedation, mechanical ventilation, or the influence of other mind-altering drugs. Even testing for the presence of a relative afferent pupillary defect may not be possible or reliable in some patients due to nonreactive pupils frequently encountered in patients with associated traumatic brain injury, coma, or raised intracranial pressures. In this background, our results may have clinical implications when radiologists who evaluate DWI sequences while reviewing brain MR imaging studies also evaluate the optic nerve for signal abnormality and, if it is present, alert the referring clinicians to the possibility of TON.

Our study has several limitations. First, it has a retrospective design. Second, the small study population may limit the generalizability of the findings. Third, inadequate spatial sampling due to a section thickness of $5 \mathrm{~mm}$ may have underestimated the incidence of diffusion restriction by precluding the identification of hyperintense signal in some patients, potentially lowering the sensitivity. DWI was performed at a 5-mm section thickness in our patient cohort. Thin sections (eg, $3 \mathrm{~mm}$ ) may improve the sensitivity. ADC measurement of the $\mathrm{ON}$ is challenging due to the small diameter of the ON because there is the potential of partial volume averaging with surrounding CSF, fat, and osseous structures. Finally, correlations between low ADC values and poor VA should be interpreted with caution because our results were based on patients with varying times from injury to MR imaging examination, which would influence the measured $\mathrm{ADC}$ values due to the ADC normalization process. However, the limitations should not negate the conclusion that ON hyperintensity due to diffusion restriction on DWI was seen in approximately one-fourth of our patients with TON and is usually associated with the worst VA (no light perception) at presentation.

\section{CONCLUSIONS}

Hyperintensity of the $\mathrm{ON}$ due to diffusion restriction after trauma is an imaging marker of TON, and when paired with reduced ADC values in the posterior segment of the $\mathrm{ON}$, it may provide important information regarding VA at presentation and hence the future visual outcome.

Disclosures: Robert K. Shin-UNRELATED: Consultancy: Novartis; Grants/Grants Pending: Novartis, ${ }^{*}$ Biogen Idec, ${ }^{*}$ Teva, ${ }^{*}$ Comments: clinical trials (principal investigator or subinvestigator); Royalties: Wolters Kluwer, Comments: coeditor of Neurology for the Non-Neurologist. *Money paid to the institution.

\section{REFERENCES}

1. Steinsapir KD, Goldberg RA. Traumatic optic neuropathy: an evolving understanding. Am J Ophthalmol 2011;151:928-33

2. Bodanapally UK, Van der Byl G, Shanmuganathan K, et al. Traumatic optic neuropathy prediction after blunt facial trauma: derivation of a risk score based on facial CT findings at admission. Radiology 2014;272:824-31

3. Bodanapally UK, Kathirkamanathan S, Geraymovych E, et al. Diagnosis of traumatic optic neuropathy: application of diffusion tensor magnetic resonance imaging. $J$ Neuroophthalmol 2013;33:128-33

4. Lessell S. Indirect optic nerve trauma. Arch Ophthalmol 1989;107:382-86

5. Cockerham KP. Traumatic optic neuropathy. In: Lounsbury ED, Bellamy RF, Zajtchuk R, eds. Textbook of Military Medicine: Ophthalmic Care of the Combat Casualty. Washington, DC: Office of the Surgeon General at TMM publications; 2003:395-403

6. Crompton MR. Visual lesions in closed head injury. Brain 1970;93:785-92

7. Steinsapir KD, Goldberg RA. Traumatic optic neuropathy: a critical update. Compr Ophthalmol Update 2005;6:11-21

8. Anderson RL, Panje WR, Gross CE. Optic nerve blindness following blunt forehead trauma. Ophthalmology 1982;89:445-55

9. Seiff SR. High dose corticosteroids for treatment of vision loss due to indirect injury to the optic nerve. Ophthalmic Surg 1990;21:389-95 
10. Walsh FB. Pathological-clinical correlations: I. Indirect trauma to the optic nerves and chiasm. II. Certain cerebral involvements associated with defective blood supply. Invest Ophthalmol 1966; 5:433-49

11. Steinsapir KD, Goldberg RA. Traumatic optic neuropathy. Surv Ophthalmol 1994;38:487-518

12. Walsh FB, Hoyt WF. Clinical Neuro-ophthalmology. 3rd ed. Baltimore: Williams \& Wilkins; 1969:2380

13. Sarkies N. Traumatic optic neuropathy. Eye 2004;18:1122-25

14. Yu-Wai-Man P, Griffiths PG. Steroids for traumatic optic neuropathy. Cochrane Database Syst Rev 2013;6:CD006032

15. Wang BH, Robertson BC, Girotto JA, et al. Traumatic optic neuropathy: a review of $\mathbf{6 1}$ patients. Plast Reconstr Surg 2001;107: 1655-64
16. Levin LA, Beck RW, Joseph MP, et al. The treatment of traumatic optic neuropathy: the International Optic Nerve Trauma Study. Ophthalmology 1999;106:1268-77

17. Galloway NR, Tong KA, Ashwal S, et al. Diffusion-weighted imaging improves outcome prediction in pediatric traumatic brain injury. J Neurotrauma 2008;25:1153-62

18. Schaefer PW, Huisman TA, Sorensen AG, et al. Diffusion-weighted MR imaging in closed head injury: high correlation with initial Glasgow coma scale score and score on modified Rankin scale at discharge. Radiology 2004;233:58 -66

19. Yang QT, Fan YP, Zou Y, et al. Evaluation of traumatic optic neuropathy in patients with optic canal fracture using diffusion tensor magnetic resonance imaging: a preliminary report. ORL J Otorhinolaryngol Relat Spec 2011;73:301-07 